\title{
Visual and phonological components of working memory in children
}

\author{
GRAHAM J. HITCH, MICHAEL E. WOODIN, and SALLY BAKER \\ University of Manchester, Manchester, England
}

\begin{abstract}
Previous studies have shown that young children's immediate memory for a short series of drawings of objects is mediated by a visual component of working memory, whereas older children rely chiefly upon a phonological component. Three experiments investigated the hypothesis that older children rely also, but to a lesser extent, on visual working memory. Experiment 1 confirmed previous evidence that 11-year-olds' memory is disrupted by phonemic similarity of object names, but is unaffected by visual similarity of the objects themselves. However, when articulatory suppression was used to prevent phonological coding, levels of recall were sensitive to visual rather than phonemic similarity. Experiment 2 compared the effects of interpolating an auditory-verbal or a visual postlist task on memory for drawings viewed either with or without suppression. The visual task had a clear disruptive effect only in the suppression condition, where it interfered selectively with recall of the most recent item. Experiment 3 compared the effects of interpolating an auditory-verbal or a mixed-modality (visual-auditory) postlist task when subjects were not required to suppress. There was greater interference from the mixed-modality task, and this effect was confined to the last item presented. These experiments are taken as confirming the presence of a small but reliable contribution from visual working memory in 11-year-old children's recall. As in younger children, visual working memory in 11-year-olds is sensitive to visual similarity and is responsible for a final-item visual recency effect. The results also show that older children's use of visual working memory is usually masked by the more pervasive phonological component of recall. Some implications for the structure of working memory and its development are discussed.
\end{abstract}

It is well-established that there is a developmental progression in the way children store a short series of drawings of familiar objects for immediate recall. Generally, older children make greater use of active mnemonic strategies, such as subvocal rehearsal of the object names (see Kail, 1984). For example, Conrad (1971) demonstrated that older children's recall was impaired by phonological similarity among the names of items, but that younger children were insensitive to this manipulation. Results such as these have encouraged the view that older children make use of a phonological storage system that is unavailable to younger children (e.g., Hitch \& Halliday, 1983). More recently, Hitch, Halliday, Schaafstal, and Schraagen (1988) demonstrated that younger children tend to rely on visual rather than phonological coding. These results raise the question of whether older children simply make no use of visual memory codes, or whether they use visual codes in parallel with phonological storage. The

Sally Baker conducted Experiment 1 and Michael Woodin conducted Experiments 2 and 3 as undergraduate projects at the University of Manchester. We are grateful for the cooperation of schools in the City of Manchester, and to the following individuals for commenting on various matters concerning the experiments and the manuscript: Donald Broadbent, Sebastian Halliday, Susanna Miller, Peter Walker, and mem bers of an informal working memory discussion group. Michael Woodin is now at the Department of Experimental Psychology, University of Oxford, England. The address for reprints is G. J. Hitch, Department of Psychology, University of Manchester, Manchester M13 9PL, England. evidence appears to be more consistent with the former interpretation, since Hitch et al. (1988) found no significant effects of manipulations such as visual similarity and visual postlist interference on older children's memory for drawings. There was, however, some indication of an effect of visual postlist interference on older children's memory for recent items (Hitch et al., 1988, Experiment 3, Figure 4a). This trend suggests that there might be a visual component in older children's recall, but that this tends to be masked by larger effects due to the use of phonological memory codes.

Evidence from studies of adults lends some support to the idea of dual coding in immediate memory. Schiano and Watkins (1981) compared immediate memory for nameable pictures and visually presented words. They observed comparable effects of phonemic similarity, word (name) length, and articulatory suppression on recall, as would be expected given the use of phonological memory codes for both types of material. In addition, however, pictures gave rise to slightly higher overall levels of recall. Schiano and Watkins attributed this difference to the greater availability of visual memory codes for pictures, in accordance with Paivio's (1971) dual coding hypothesis. A problem for such an interpretation, however, is that the superiority of pictures might be due to some other factor, for example, the possibility that pictures may allow faster access to semantic codes (Potter \& Faulconer, 1975). 
The main aim of the present series of experiments was to test directly the hypothesis that there is a visual component in older children's memory for a series of drawings, in addition to the much greater phonological component. In order to reveal the presence of visual codes, it was considered necessary to test for them initially under conditions in which the tendency to rely on phonological representations was disrupted. Our first two experiments made use of the technique of articulatory suppression, since there is considerable evidence that suppression disrupts the use of phonological memory codes in adults (see, e.g., Baddeley, 1986) and in older children (see, e.g., Hitch \& Halliday, 1983). Based on the assumption that visual coding effects are normally masked by effects of phonological coding, we expected to obtain clear evidence for the use of visual coding only under conditions of articulatory suppression.

Positive results would be methodologically important, since the investigation of visual memory in adults (presumed here, incidentally, to behave similarly to that in older children) is often hampered by unwanted effects associated with the use of phonological memory codes. Some investigators have resorted to using highly artificial stimulus materials that are difficult to encode verbally (e.g., Phillips \& Christie, 1977), whereas others have used articulatory suppression to block phonological coding (e.g., Broadbent \& Broadbent, 1981). There is, however, little direct evidence that the codes supporting memory under suppression are visual. The present experiments should help to clarify this point.

The outcome is also of interest in the context of attempts to model short-term memory and its development. Hitch et al. (1988) interpreted their results in terms of the working memory model of Baddeley and Hitch (1974), which distinguishes between separate subsystems that deal with visuospatial and phonological representations. According to this model, visual materials are fed automatically into the visuospatial store, but the control process of subvocal articulation is necessary for their names to be held in the phonological storage system. Thus the working memory model leads us to expect both a visual and a phonological component in older children's immediate recall of visually presented materials. Indeed, it was also this model that led us to predict that articulatory suppression would reveal the presence of the visual component by blocking access to the phonological storage system (see above).

From a developmental perspective, the progression suggested here is one of a proliferation of memory codes whereby phonological coding complements rather than replaces visual coding. This is consistent with models of general cognitive development that propose a maturational increase in the diversity of mental representations (see, e.g., Kosslyn, 1980, p. 408). Finally, the identification of a visual component in older children's recall would allow some useful comparisons with visual working memory in younger children.

\section{EXPERIMENT 1}

This experiment compared memory for visually similar drawings, drawings with phonemically similar names, and control items in 11-year-old children either with or without articulatory suppression. A younger (5-year-old) group of children was also tested on memory for the same materials without suppression in order to include a replication of the basic visual similarity effect. Our previous demonstrations of visual similarity effects in younger children (Hitch et al., 1988) are open to the possible objection that the visually similar materials were more semantically similar than the control items. We therefore used in the present study materials that did not come from a common category. We again included checks on young children's accuracy in identifying the materials, to rule out the possibility that the visual similarity effect is due to difficulty in initial encoding. It was considered unnecessary to check older children in this way.

\section{Method}

Subjects. There were three groups of 18 subjects, all of whom attended an urban primary school in South Manchester. The 5-yearold group ( 8 boys, 10 girls) had a mean age of 5 years, 3 months (range $=4,9-5,8$ ); the 11-year-old control group ( 9 boys, 9 girls) had a mean age of 10,11 (range $=10,4-11,3$ ); and the 11-yearold suppression group ( 9 boys, 9 girls) had a mean age of 10,8 (range $=10,4-11,2)$.

Materials. The experimental materials comprised three sets of eight line drawings of common objects mounted on $15 \times 10.5 \mathrm{~cm}$ cards (see examples in Figure 1). The names of the items were highfrequency, monosyllabic nouns, with word frequency (Thorndike \& Lorge, 1963) equated across sets. Items in the visually similar set had similar elongated outlines and were depicted in the same oblique angle of orientation. They had phonemically dissimilar names: key, spoon, pen, watch, tie, brush, bone, and spade. Items in the phonemically similar set were visually dissimilar from one another but had names that rhymed: bat, cat, mat, hat, map, rat, tap, and cap. Items in the control set were neither visually nor phonemically similar to one another: chair, shoe, fork, bell, tree, bird, kite, and door. There was also a small set of control items for use in the practice phase of the experiment. Pilot testing had shown that, when asked to name these items, most children readily supplied the designated response.

Design. Type of materials was manipulated as a within-subjects factor, such that there were six trials for each type of material per subject. Trials were blocked by type of material, and the order of blocks was fully balanced across subjects. A trial consisted of a sequence of five items for 11-year-olds and three items for 5-yearolds. These sequence lengths were chosen so as to avoid floor and ceiling effects within each age group. Sequences were constructed afresh for each subject by random sampling without replacement from each of the three sets of materials. The 11-year-old children were assigned at random to either a control or an articulatory suppression group.

Procedure. Each child was tested individually in school. Testing began by showing a child all the drawings that were to be presented and requiring each one to be named. Any failures to produce the designated name were corrected. This procedure was repeated until all the items were named correctly; one reperition was sufficient. 


\section{Control}
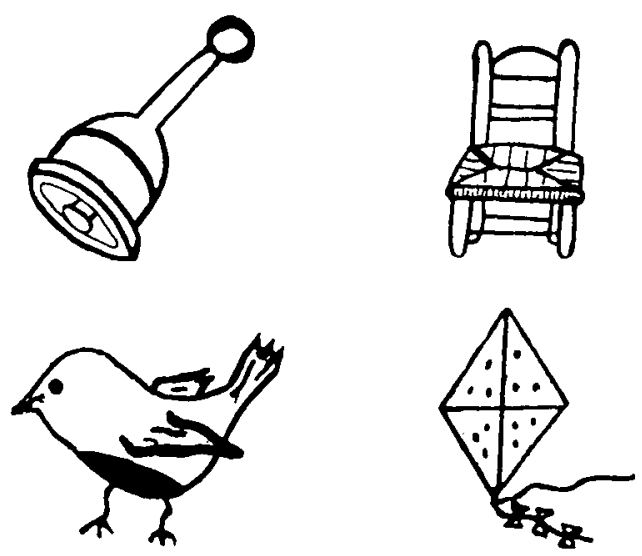

Visually similar
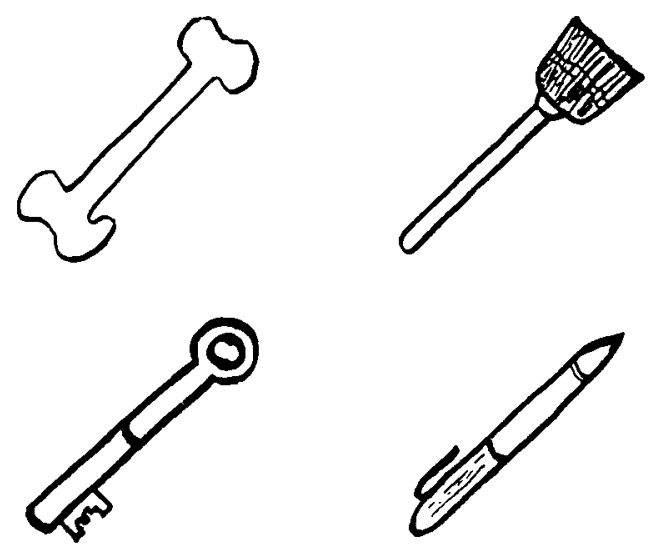

Phonemically similar names
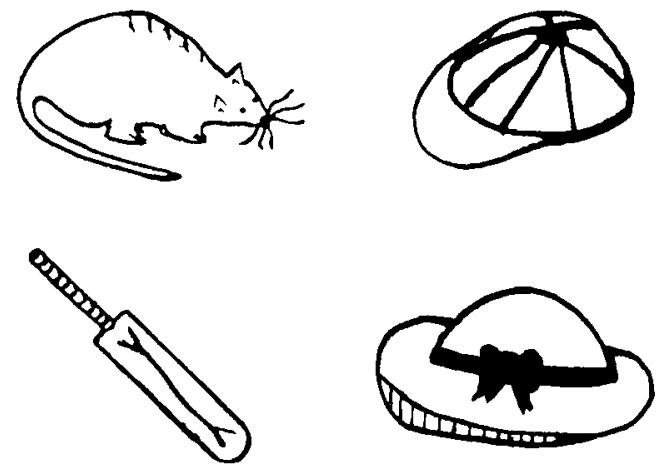

Figure 1. Sample of materials used in Experiment 1.

In the next phase, the children were given practice on the memorization task using shorter sequences than would occur in the experiment proper. Younger children were shown sequences of two items, older ones four. The subjects were asked to play a game in which they looked away while the experimenter laid out the memory items. These were put face up in a horizontal row, and each was covered with a blank card. The importance of remaining silent during sequence presentation was stressed to both the younger children and the 11-year-old controls. Children in the articulatory suppression group were required to start saying aloud the word "the" when the first drawing was shown and to stop only when the last item was covered up. They did this at a rate of approximately two repetitions per second. The subject was invited to watch while the experimenter removed and then replaced each cover in turn; presentation was synchronized with an auditory-visual metronome set at a 2 -sec rate. Immediately after showing the last item, the experimenter pointed to the cover at the first location and asked the child to say the name of the picture underneath. Prompting was continued in this fashion for all items. The subjects were encouraged to say that they did not know whenever they were uncertain. Older children were given two of these practice trials with four cards, followed by an additional one or two practice trials with sequence length increased to five. Younger children were given from two to four such trials with two items, depending upon how quickly they grasped the procedure, followed by a final one or two practice trials with three-item sequences. At the end of training, it was possible for the experimenter to run smoothly through the presentation and prompting phases of each trial without needing to give the subject any verbal instructions.

For the two older groups, the experimental trials followed immediately after the practice phase, and the entire test session lasted about $25 \mathrm{~min}$. Because of possible problems of fatigue, younger children were treated slightly differently. They were tested in two sessions, one in the morning and the other in the afternoon, each lasting about $15 \mathrm{~min}$. The first session ended with completion of the first block of experimental trials. The second session began with all the drawings being shown and renamed by the child, followed by two trials of practice on the memory task, followed by the remaining two experimental blocks of six trials. The procedure on each trial was also slightly different in that the younger children were shown the items they failed to recall correctly and were asked to rename them before going on to the next trial. This was an attempt to ensure that children used the designated names for the items. The session ended with all the drawings being shown a final time for a further renaming.

\section{Results}

Performance was scored as the number of items correctly recalled at each serial position. Table 1 summarizes the observed means and standard deviations. Because the younger children were given a different list length, it was considered appropriate to analyze their data separately.

A one-way analysis of variance showed that type of material had a significant effect on number of items recalled by the 5-year-olds $[F(2,34)=24.84, p<.01]$. Planned comparisons showed that visually similar materials were less well recalled than control materials $[F(1,17)=33.6, p<.01]$, whereas there was no difference between phonemically similar materials and control items. Analyses of the accuracy with which the 5-yearolds could name the items gave no support to the view that their poorer recall of visually similar items was due to errors that occurred at initial encoding. Children made virtually no errors when, at the end of each trial, they were asked to name any items that had not been correctly recalled. There were only 6 such errors in 402 opportunities, none of which was associated with the visually similar materials. There were also very few errors on the naming posttest, and no indication whatsoever that such errors were associated with the visually similar materials.

Data from the 11-year-olds were subjected to a mixed two-way analysis of variance, with materials as the within- 
Table 1

\begin{tabular}{|c|c|c|c|c|c|c|c|}
\hline & \multirow[b]{3}{*}{$n$} & \multicolumn{6}{|c|}{ Type of Materials } \\
\hline & & \multicolumn{2}{|c|}{ Control } & \multicolumn{2}{|c|}{$\begin{array}{l}\text { Visually } \\
\text { Similar }\end{array}$} & \multicolumn{2}{|c|}{$\begin{array}{l}\text { Phonemically } \\
\text { Similar } \\
\end{array}$} \\
\hline & & $M$ & $S D$ & $M$ & $S D$ & $M$ & $S D$ \\
\hline 5-Year-Olds & 18 & 1.90 & 0.49 & 1.41 & 0.54 & 1.97 & 0.53 \\
\hline 11-Year-Olds Control & 18 & 3.64 & 0.96 & 3.43 & 1.08 & 2.71 & 0.70 \\
\hline 11-Year-Olds Suppression & 18 & 2.07 & 0.68 & 1.70 & 0.78 & 2.00 & 0.57 \\
\hline
\end{tabular}

subjects factor and the presence or absence of articulatory suppression as the between-subjects factor. Suppression had a large disruptive effect on the number of items recalled $[F(1,34)=35.8, p<.01]$, and there was also a significant effect of type of materials $[F(2,68)=6.99$, $p<.01]$. Of particular interest is the interaction between the effects of suppression and type of materials $[F(2,68)$ $=8.44, p<.01]$. A series of planned comparisons against performance on the control materials showed that in the absence of suppression, recall was disrupted by phonemic similarity $[F(1,17)=23.7, p<.01]$ and was unaffected by visual similarity $(F<1)$. Under suppression, however, recall was disrupted by visual similarity $[F(1,17)$ $=6.52, p<.05]$, but not by phonemic similarity $(F<1)$.

\section{Discussion}

The results of the experiment are clear-cut and in line with expectations. First, they confirm Hitch et al.'s (1988) finding that young children's immediate recall is poorer for visually similar materials, suggesting that they retain the items in some form of visual storage. The complete absence of any disruption due to phonemic similarity in this group suggests that they make no use of phonological storage, despite the requirement for verbal recall. This last finding represents a minor discrepancy with the observation of a small but reliable effect of word length in children of this age (Hitch et al., 1988) and clearly merits further investigation.

The key findings, however, concern the effects of suppression on 11-year-olds. When these older children were not suppressing, their recall was poorer for phonemically similar materials. This effect is expected, given active rehearsal of the names of the materials in the phonological component of working memory, and is consistent with previous data (Conrad, 1971; Hitch et al., 1988). Suppression lowered the overall amount of material recalled, and also removed the effect of phonemic similarity. This too is consistent with previous results (Hitch \& Halliday, 1983 ) and with the hypothesis that suppression interferes selectively with use of phonological working storage. Most importantly, however, suppression led to a significant visual similarity effect, suggesting that, in these circumstances, recall was based on visual storage. Further- more, inspection of Table 1 shows that when 11-year-olds engaged in articulatory suppression, they behaved very similarly to 5-year-olds, both in terms of absolute numbers of items recalled and the effects of type of materials.

Thus, our results confirm the prediction that older children would show evidence of using visual short-term storage when a phonological coding strategy is disrupted. On the other hand, the data provide no evidence for the presence of a visual component in recall when the phonological coding strategy is available to subjects.

\section{EXPERIMENT 2}

Our next experiment provided a further, independent test of older children's tendency to use visual working memory when phonological coding is disrupted by articulatory suppression. Our previous evidence for visual working memory in younger children (Hitch et al., 1988) included the existence of a visually based recency effect in a task involving recall of a series of drawings in reverse temporal order. This visual recency effect was largely confined to the final list item, a finding that corresponds with evidence for single-item recency in memory for nonnameable visual materials in adults (Phillips \& Christie, 1977). The visual basis of our recency effect in young children was revealed by its sensitivity to the modality of a postlist task, such that a visual task was more disruptive than an auditory-verbal task. We also showed that under the same experimental conditions, older children were more sensitive to an auditory-verbal postlist task. In the present investigation of older children, we therefore manipulated both the presence/absence of articulatory suppression during presentation of the drawings and the modality of a postlist task. In the absence of suppression, we anticipated simply repeating our previous observation of greater disruption from an auditory-verbal postlist task. However, if under articulatory suppression older children behave more like younger children and rely more heavily upon visual working memory, the effects of postlist interference should then conform to a different pattern. Specifically, a visual postlist task should be more disruptive. Moreover, we anticipated that under suppression the effect of the visual task should be to disrupt final-item recency. This would follow from the proper- 
ties of the visual memory system as it appears in younger children.

An earlier study of adult subjects is highly relevant to the present investigation. Martin and Jones (1979) examined the effects of auditory or visual postlist interference on free recall of visually or auditorily presented word lists. In one experiment, they found that the auditory postlist task reduced the recency effect more than the visual task did, even when the words were visually presented. This is consistent with our previous data on older children's recall of drawings (Hitch et al., 1988). In another experiment, Martin and Jones (1979) required their adult subjects to perform articulatory suppression during the visual presentation of word lists. Under these conditions, the recency effect was reduced more by visual than by auditory postlist interference. These findings were interpreted in terms of subjects' greater dependence on visual storage under articulatory suppression. However, Martin and Jones did not control order of report. The importance of doing so is emphasized by Dalezman (1976), who showed that a substantial part of the recency effect in free recall is dependent upon subjects' reporting the last items first. The effects reported by Martin and Jones might therefore reflect changes in recall order associated with the different postlist tasks, rather than the subjects' ability to recall the final items per se. By strictly controlling order of report, the present experiment avoids this serious difficulty of interpretation. A further problem with Martin and Jones's study is that their "visual" postlist task involved solving an anagram, and therefore had a large verbal component. The present study avoids this difficulty by using a simple pattern matching task.

\section{Method}

Subjects. Thirty-six children ( 21 girls and 15 boys) were recruited from three schools serving inner-city areas of Manchester. Their average age was 10,9 (range $=10,3-11,2$ ). They were randomly assigned to two equal-sized groups.

Materials. Three sets of stimulus cards measuring $9 \times 9 \mathrm{~cm}$ were made. Two of these constituted the to-be-remembered sets, in which each card bore a line drawing of a common object copied from the Peabody Picture Vocabulary Test (Dunn, 1965). Items were selected such that the objects were visually dissimilar from one another and had phonemically dissimilar, monosyllabic names. It was established through pilot testing that children could readily name the items. There were five items in the practice set (brush, bus, knife, sock, and tank) and 20 items in the experimental set (ball, bell, bike, car, chair, cot, cup, drum, glove, gun, hut, kite, lamp, pin, purse, ring, sink, skirt, spoon, and tin). The final set of cards was used in the visual postlist task. There were nine cards in this set, each bearing a $3 \times 3$ matrix. There were three designs (see Figure 2), each mounted on three cards.

Design. A mixed design was used, with presence or absence of articulatory suppression manipulated between subjects and source of retroactive interference manipulated within subjects. There were three types of retroactive interference: visual, auditory-verbal, and none. The visual and auditory-verbal tasks were selected so as to be well within the subjects' capabilities and to each take approximately $4 \mathrm{sec}$ to complete.

Each subject was given one block of three five-item lists in each retroactive interference condition. The order of blocks was systematically rotated across subjects. The word lists were constructed
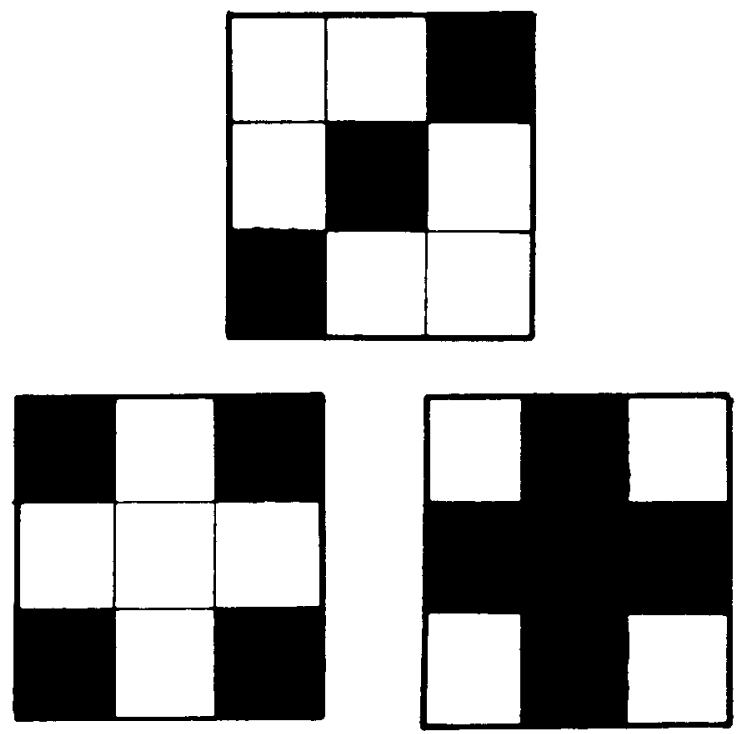

Figure 2. Matrices used in the visual matching task in Experiment 2.

by random sampling from the experimental set such that no item was repeated within any block. Each subject was given freshly randomized lists.

Procedure. The subjects were tested individually. They were first asked to name each stimulus card in the experimental set in order to become familiar with the materials and the standardized name for each item.

The procedure was then explained using the practice set. Cards were shown at a rate of one item every $2 \mathrm{sec}$ at separate locations in a left-to-right row on a table in front of the subject. Each card was turned over to lie face down after its presentation. The experimenter cued the presentation of each list with the word "ready."

Subjects assigned to the irrelevant articulation group were additionally trained to say "bla-bla-bla- ..." during the presentation procedure, at a rate of about 2-3 blas per second. This task was not thought to differ in any significant way from that used in Experiment 1 . Subjects in the control group were given no special instructions as to what to do during presentation.

Following training on the presentation procedure, the subjects received training on the postlist task (if any) for the experimental condition to be presented first and on the recall procedure. The latter was the same in all three conditions. The experimenter prompted verbal recall by pointing to each card in turn, starting with the most recent item. Although there was a certain amount of verbal prompting during training of the recall procedure, the experimenter remained strictly silent during this part of the experimental trials.

Recall followed presentation immediately when there was no postlist task, and by approximately $4 \mathrm{sec}$ when there was one. The auditory-verbal task consisted of the experimenter's reading aloud a set of three random letters, which the subject had to repeat back immediately. The experimenter then presented a second triad for immediate repetition. Letter combinations that spelled words were avoided. The visual postlist task made use of the third set of stimulus cards. One of each of the three different matrix designs was placed face up in a line adjacent to the location in which the experimental lists were presented. This line extended away from the subject and remained in place throughout the testing session. The remaining six cards were placed face down in a randomly ordered pile adjacent to the line. The task consisted of the subject's taking the top card from the pile and placing it face up on top of the corresponding card in the line. Subjects in the articulatory suppres- 
sion condition were required to continue to suppress throughout this task. Pilot work had suggested that this was necessary to prevent subjects from starting to rehearse the names of the items. Any such recoding would, of course, obviate the purpose of the experimental manipulation. For each postlist task, the subject was first given training on the interference task alone before being given practice in the complete procedure for each trial. The three experimental trials for each condition were started only when the experimenter judged that the procedure was fully understood. Procedural variations associated with the different retroactive interference conditions were introduced only immediately before the start of the relevant block of trials. The experimenter recorded each item recalled as correct only when the subject's response was the item that had been presented at the prompted location.

\section{Results}

As expected, the subjects performed the postlist tasks without error. Figure 3 shows serial position curves for recall in the various conditions of the experiment. It is clear that the requirement for articulatory suppression changed the pattern of postlist interference effects in a dramatic way. This was confirmed by a three-way analysis of variance, with factors of articulation, postlist interference, and serial position. There were significant main effects of serial position $[F(4,136)=19.5, p<.01]$ and postlist interference $[F(2,68)=14.3, p<.01]$, but not of articulatory suppression $[F(1,34)=3.39]$. All the interactions in this analysis were also significant, except that between articulation and serial position. Separate analyses of variance were carried out for both articulation groups in order to clarify these results.

For subjects who were not required to perform articulatory suppression, the curves in all three conditions had a broadly similar shape, with both primacy and recency

(a)

\section{Control Condition}

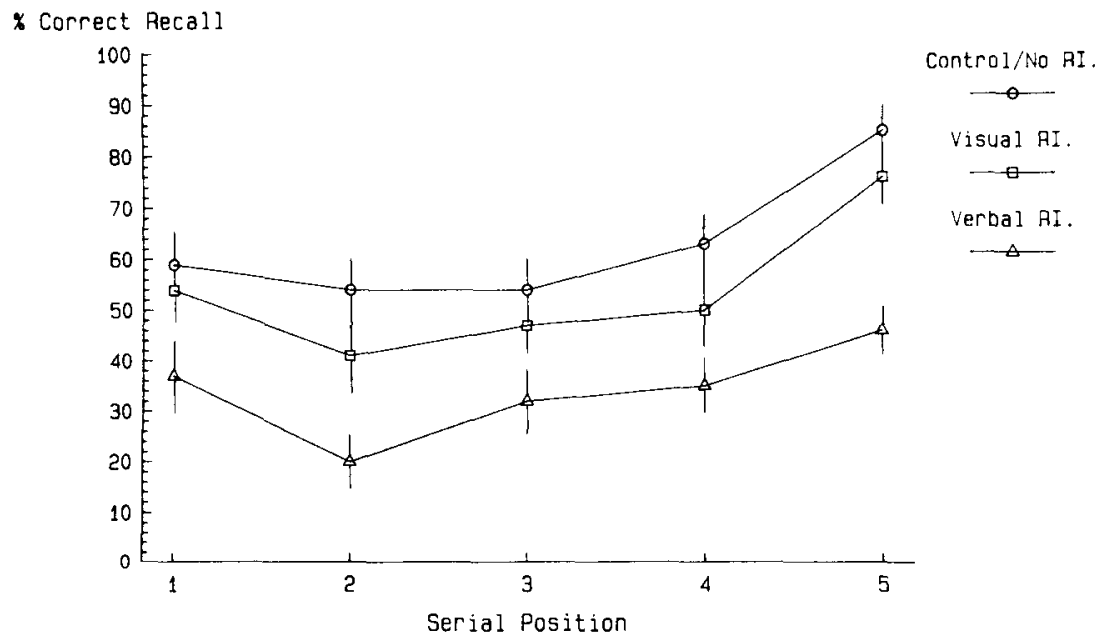

(b)

\section{Suppression Condition}

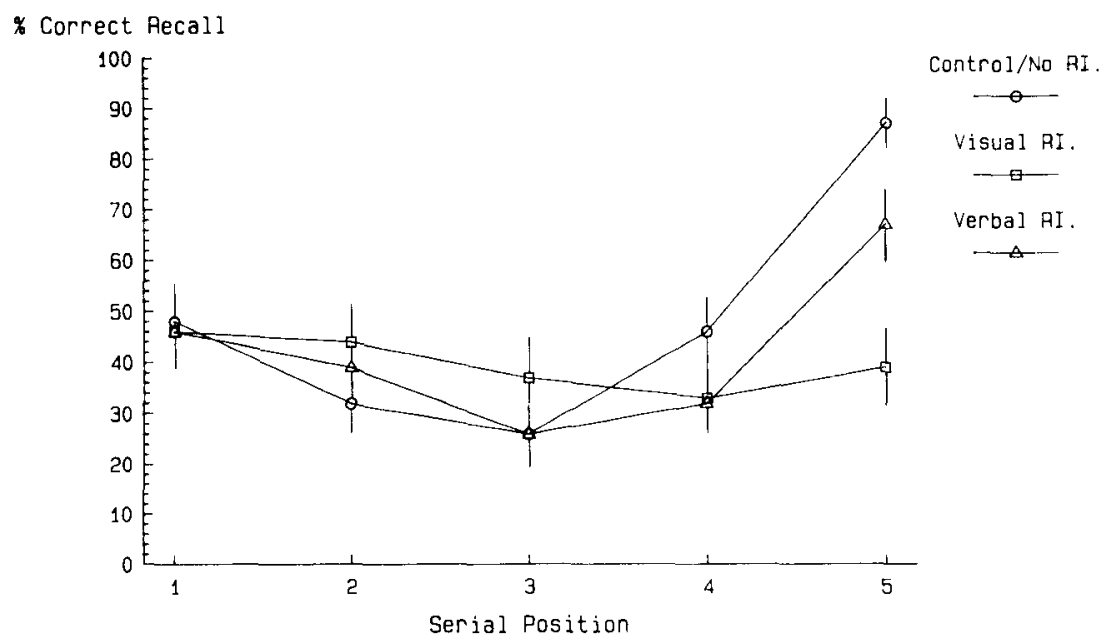

Figure 3. Serial position curves from Experiment 2 showing the effects of an auditory -verbal and a visual postlist task on 11-year-olds' memory for drawings presented with either (a) no concurrent activity or (b) articulatory suppression. $\mathbf{R I}=$ retroactive interference. 
effects in evidence. A two-way analysis of the data from this group confirmed significant effects of serial position $[F(4,68)=8.18, p<.01]$ and postlist interference $[F(2,34)=15.6, p<.01]$, but no interaction $(F<1)$. A series of Tukey tests (with $\alpha$ set at .05) showed that recall was poorer following the auditory-verbal task than either the visual task or no task, and that the small observed difference between the latter conditions was nonsignificant.

For subjects who had to suppress during presentation of the memory items, the serial position curves look rather different. Serial position was again highly significant $[F(4,68)=12.2, p<.01]$, but there was no main effect of postlist interference $[F(2,34)=2.23]$. The interaction was, however, highly significant $[F(8,136)=3.29, p<$ $.01]$. It is clear by inspection that this interaction derives from the differential effectiveness of the two types of postlist interference in reducing recency. This was confirmed by a significant simple main effect of interference on recall of the final item $[F(2,34)=10.5, p<.01]$, but no such effect when data from serial positions 1-4 were pooled. Tukey tests showed that recall at the last position was significantly poorer following the visual task than in the control condition, but that the auditory-verbal task had no significant effect. The difference between the two tasks just failed to reach significance.

In summary, the results show that in the absence of articulatory suppression, auditory-verbal postlist interference disrupts recall, whereas visual interference has only a small, unreliable effect. The effect of auditoryverbal postlist interference is spread through all serial positions. When articulation is suppressed, effects of postlist interference are confined to the final serial position. In this case, visual interference disrupts recall, whereas the effect of verbal interference is small and unreliable.

\section{Discussion}

Although the results of this experiment are complicated, the main hypothesis is clearly supported: older children's recall of nameable drawings is disrupted by an auditoryverbal postlist task when they do not have to perform articulatory suppression, but a visual task is the more disruptive when they do suppress. When the use of phonological storage is prevented, such children are evidently reliant on visual working memory for remembering the items they have been shown.

These results independently confirm the conclusion drawn from Experiment 1. They are also consistent with the outcome of Martin and Jones's (1979) investigation of adult subjects' memory for visually presented words under suppression. We can suggest that Martin and Jones's failure to control order of report was probably not important. We would maintain, however, that our use of a simpler, nonverbal postlist task provides a more compelling demonstration.

The detailed nature of the present results is particularly interesting. First, we find that when children did not suppress, the effects of the visual postlist task were small and nonsignificant. This is consistent with the absence of a significant effect of visual similarity for older children who were not suppressing in Experiment 1. Thus the two experiments agree in suggesting that visual coding effects are either absent or overshadowed in older children when phonological working memory is available. Experiment 3 explores which of these two interpretations is correct.

A second interesting feature of the present results is the evident difference in the serial positions affected by the visual and auditory-verbal postlist tasks. In the absence of suppression, the auditory-verbal task has a large and clear-cut through-list effect (although there is some suggestion that there may be additional interference with final items, see Figure 3a). This through-list effect is similar to the effect of articulatory suppression on immediate recall. Indeed, there is very little effect of the auditoryverbal postlist task on memory for lists presented under suppression (what little effect there might be is confined to more recent items; see Figure 3b). We interpret these data as confirming the massive contribution of phonological storage to older children's recall of items from all serial positions under normal conditions. In contrast, the only significant effect of the visual postlist task is on memory for the most recently presented item under suppression. We interpret this as confirming the much smaller part that is normally played by visual working memory in older children's recall. We note also that the restriction of the visual recency effect to the final item in older children engaging in suppression corresponds with the size of this effect in younger children (Hitch et al., 1988) and with comparable adult data (Phillips \& Christie, 1977).

\section{EXPERIMENT 3}

Experiments 1 and 2 demonstrated that older children use visual working memory when the requirement for articulatory suppression prevents the use of phonological storage. Experiment 3 tests for the possibility that there is a visual component to recall in the absence of articulatory suppression. At issue here is whether the results of the previous experiments should be interpreted as revealing a visual component of recall that is present in the absence of suppression, or in terms of a switch of encoding strategy induced by suppression.

From the outset, it was anticipated that any visual component would be small given the extent to which such subjects rely upon phonological representations. The results of Experiment 1 suggested that the visual similarity effect, at least as manipulated in that experiment, would not be sufficiently sensitive to detect the use of visual working memory. Although older children who were not engaging in articulatory suppression did show a slight tendency to remember fewer visually similar items (alongside the much larger effect of phonemic similarity), this small difference did not approach significance. We opted to continue to investigate the recency effect in backward recall, for two reasons. First, it seems that recency is highly sensitive to the modality of postlist interference. Second, one 
detailed aspect of the results of Experiment 2 suggests that there might indeed be both phonological and visual components of recency. Inspection of Figure 3a suggests that, in the absence of articulatory suppression, the auditoryverbal postlist task did not entirely abolish recency. This, in turn, suggests that the remaining component of recency may be based on visual memory codes. If so, we reasoned that postlist interference, which combined both visual and auditory-verbal tasks, would be more effective at reducing recency than an auditory-verbal task alone, since a combined task ought to disrupt both components of the recency effect. Experiment 3 tested this prediction.

An important methodological problem in designing the study was to avoid biasing the results by using interfering tasks of unequal difficulty. In the previous experiment, we had found a clear crossover by contrasting the matching of one matrix pattern with the repetition of two threeletter lists. These tasks seemed easy for children to perform with $100 \%$ accuracy and took the same time to complete. We therefore reasoned that a combined task involving matching one matrix and then repeating one list of three letters (or vice versa) would take about the same time as one involving the repetition of three lists of letters. Furthermore, both tasks ought to be easy for children to perform without error. Pilot work confirmed our reasoning, so this procedure was adopted.

\section{Method}

Subjects. Eighteen children ( 9 girls and 9 boys) were recruited from two inner-city primary schools in Manchester. Their average age was 11,2 (range $=10,11-11,5$ ).

Materials. This experiment used the same three sets of stimulus materials as in Experiment 2.

Design. The experiment used a within-subjects design with two independent variables, postlist interference (control, auditoryverbal, and combined visual and verbal) and serial position. Each subject was tested for recall of six five-item lists under each of the three levels of postlist interference.
There were two test sessions, each comprising a block of three lists in each interference condition. The order of blocks was rotated factorially across subjects, and different orders were used in the two sessions for each individual subject.

The visual and auditory-verbal components of the combined postlist interference task were performed sequentially. The order of these two components was counterbalanced both across sessions within subjects and between subjects.

Procedure. The familiarization, training, presentation, and recall procedures all followed exactly the procedure adopted in Experiment 2 for the group of subjects who were not required to perform articulatory suppression. The only differences concerned the nature of the postlist interference tasks.

In the control condition, recall once again followed immediately after list presentation. In the auditory-verbal condition, subjects were again asked to repeat sets of three orally presented letters immediately after list presentation and prior to recall. This time, however, three such lists were presented, and the typical duration of the task was 6-8 $\mathrm{sec}$. In the combined interference condition, subjects were required to match one matrix card with its pair, in precisely the same way as in Experiment 2 . In addition, they had to repeat a single list of three orally presented letters. The combined task was also interpolated between list presentation and recall, and also lasted typically 6-8 sec.

\section{Results}

Performance on each of the postlist tasks was errorfree. In the memory task, items were scored correct only if they were recalled in the correct position. Figure 4 shows the serial position curves obtained for the three experimental conditions. It is immediately evident that the combined postlist task was the more effective at removing recency for the final item, even though it seemed to have less effect on recall of earlier items than did the auditory-verbal task.

A two-way analysis of variance revealed significant main effects of condition $[F(2,34)=25.5, p<.01]$ and serial position $[F(4,68)=17.8, p<.01]$. Moreover, the interaction between condition and serial position was also significant $[F(8,136)=5.82, p<.01]$. When serial po-

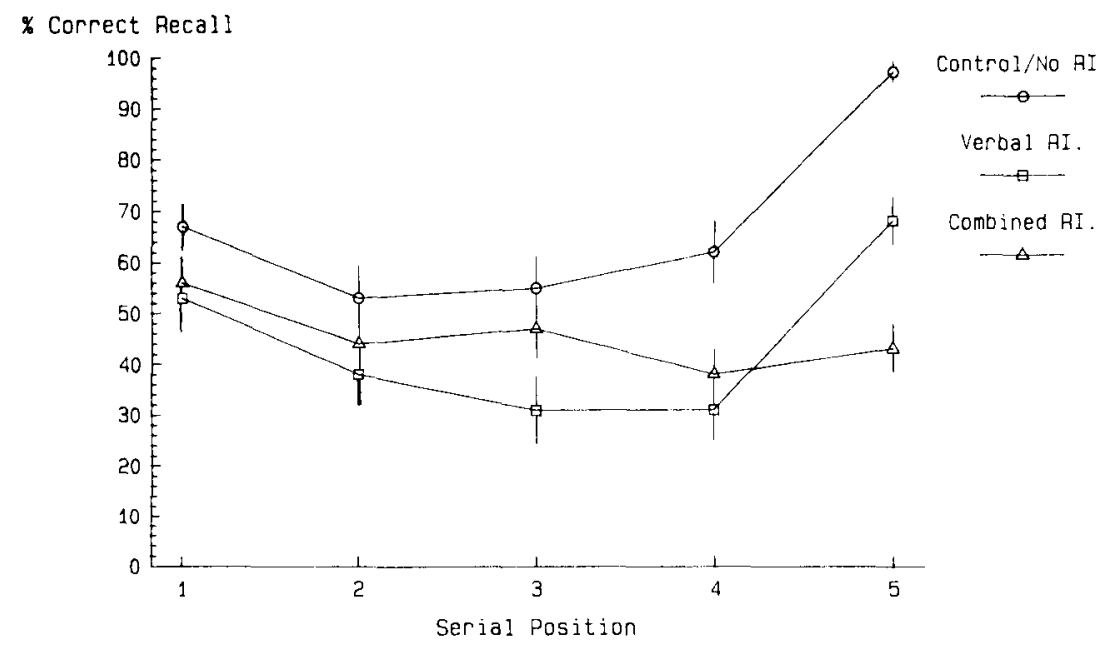

Figure 4. Serial position curves from Experiment 3 showing the effects of an auditory-verbal postlist task and a combined-modality task (visual/auditory-verbal) on 11-year-olds' memory for drawings. $\mathbf{R I}=$ retroactive interference. 
sitions 1-4 were analyzed separately, however, the interaction disappeared $[F(6,102)=1.49]$. A series of Tukey tests was carried out to explore differences among conditions over positions 1-4. These showed that recall was higher in the control condition than in either interference condition. The difference between the two interference conditions, although consistent across positions, was not significant. A parallel series of Tukey tests on recall of the final list item revealed that recall was again significantly higher in the control condition. In this case, however, recall following combined interference was significantly poorer than that following auditory-verbal interference.

A further analysis of data from the combined interference condition was carried out to see if the order of the two tasks was important. Although there was a slight tendency toward less recency when the visual task was performed first, the difference was not significant.

Overall, therefore, the results show that the auditoryverbal postlist task alone was insufficient to remove the recency effect associated with the final list item, and that recency was abolished only when the postlist task included a visual component.

\section{Discussion}

The greater effectiveness of the combined versus singlemodality postlist tasks in removing recency suggests that both phonological and visual memory codes contribute to recency. In other words, it seems that immediate memory for nameable pictures in older children involves dual rather than unitary coding. We infer, therefore, that the visual codes observed under articulatory suppression in Experiments 1 and 2 were not the result of suppression's inducing a switch to a developmentally more primitive encoding strategy. Instead, we support the view that suppression reveals visual coding effects that are normally masked by the predominance of phonological coding.

It is important, however, to consider the possibility that the results of the present experiment could be explained in terms of a hypothetical difference in the levels of difficulty of the two postlist interference tasks, despite our attempts to equate them. According to this view, one would have to assume that the combined task was more difficult than the single one. There are two reasons for rejecting such an interpretation. First, the effects of the two tasks were statistically indistinguishable over serial positions 1-4, with a slight tendency for better recall following the combined task. The combined task might have been expected to create more, rather than slightly less, interference had it been the more difficult. Second, a levels of difficulty interpretation provides no account of the restriction of the difference between the effects of the two tasks to the final serial position. The dual coding interpretation is superior here since, as we have seen, visual recency is thought to extend over only the last item in a sequence.

For the above reasons, we conclude in favor of dual coding. However, it is interesting to note that in the present experiment children remembered considerable amounts of information, even following the combined postlist task. This may indicate a form of visual memory that is not susceptible to interference from subsequent visual events (see Hitch et al., 1988). Some proportion of this "residual" memory alternatively may reflect unspecified coding strategies that are neither visually nor verbally based, and thus are resistant to the effects of the combined interference task.

\section{GENERAL DISCUSSION}

We began by asking whether visual coding effects might be observed in older children's recall of nameable pictures when phonological coding strategies were blocked by articulatory suppression. The results of the first two experiments present unequivocal support for this notion by demonstrating the presence of a visual similarity effect (Experiment 1) and a one- (or possibly two-) item visual recency effect under suppression (Experiment 2). Neither of these effects could be clearly observed when subjects were free to rehearse picture names during presentation. Our final experiment suggested that the reason for the apparent absence of these effects under such conditions may be that phonological codes normally dominate in recall, thus effectively masking the use of visual representations. Given sufficiently sensitive measures, it proved possible to show that even when subjects are free to rehearse picture names, visual coding contributes to recency for the final picture seen. This was achieved by demonstrating that a mixed-modality postlist task was more effective in reducing recency than a solely auditory-verbal task. We conclude, therefore, that when older children are presented with nameable pictures for immediate recall, both visual and phonological memory codes are established. We conclude also that the functional effect of articulatory suppression is to "subtract" the phonological component from recall, rather than to induce a switch to an encoding strategy that would not otherwise be utilized. In developmental terms, therefore, our data support the view that there is a proliferation of memory codes such that, although phonological coding becomes massively important in older children, it does not entirely replace the type of visual coding that is also seen at an earlier stage.

This general pattern of results can be readily interpreted in terms of the working memory model (Baddeley, 1986; Baddeley \& Hitch, 1974), first put forward to account for adult behavior. Thus, the effects of articulatory suppression on older children were predicted from a direct application of this model, in that suppression is thought to interfere selectively with the phonological component of working memory, leaving the visual subsystem unaffected. The model also accounts neatly for the difference between younger and older children if the reasonable general assumption is made that processes that are obligatory (automatic) in adults will develop earlier than control processes. We suggest that at around 5 years of 
age, visual inputs gain obligatory access to the visual component of working memory, as in adults, but that the control processes necessary for them to gain access to the phonological storage component have yet to develop. This interpretation also leads us to expect that spoken inputs will behave differently and will gain access to phonological storage even in very young children, since this process is held to be obligatory in the adult model (Baddeley, 1986). This prediction has been borne out (Hitch \& Halliday, 1983), suggesting that the model does indeed capture some important developmental regularities.

One aspect of performance is not satisfactorily explained by the current working memory model, however. This is the "residual memory" evident when neither the phonological nor the visual subsystem seems likely to contribute much to recall, mentioned in the discussion of Experiment 3. Residual recall of this sort is clearly ubiquitous, and is apparent in both of the other experiments as well (above-chance recall of visually similar drawings encoded under suppression in Experiment 1; and recall of drawings encoded under suppression and followed by a visual postlist task in Experiment 2). In the working memory model, retention in these conditions must be attributed to a poorly understood third subsystem (the "central executive"'; Baddeley, 1986; Baddeley \& Hitch, 1974), or possibly to long-term storage. Alternative theoretical approaches may fare rather better here. For example, Drewnowski's (1980) model of short-term memory postulates that recall involves the retrieval of several types of stored attribute, rather than output from the limited number of subsystems that make up working memory. His model specifies the attributes that should form the basis of residual memory in our experiments, and could be used to make testable predictions. It is not unique in this respect, since some other multicomponent models (see, e.g., Barnard, 1985; Monsell, 1984) also make explicit claims about this aspect of recall. In all cases, however, the various predictions remain to be properly tested and evaluated. We note also that our main findings concerning visual and phonological coding are inconsistent with some aspects of these models. For example, Drewnowski (1980) did not identify a visual attribute and assumed that the encoding of all attributes is automatic. However, since our experiments did not aim to choose among alternative models, it is inappropriate to pursue this matter any further here. We can, nevertheless, be confident that the important theoretical questions concern what sorts of multicomponent model should be entertained, since the simpler, but quite common, assumption that short-term memory is an undifferentiated resource can evidently be ruled out. We turn now to consider what light our results shed on the nature of the visual component of immediate recall, using for convenience the terminology of the working memory model.

The results suggest some tentative conclusions. First, it is evident that the visual component of recall in older children is, so far as we can tell, indistinguishable from the proportionately much larger visual component observed when younger children are tested under the same conditions. Specifically, closely comparable effects of visual similarity and a visual recency effect confined to the last item are found both in young children and in older children engaging in articulatory suppression. These detailed correspondences are impressive. We have suggested that the visual working memory system stores information about visual features (e.g., size, shape, and orientation) and recency of occurrence (Hitch et al., 1988). According to this interpretation, the visual similarity effect is seen as reflecting the forgetting of stored features, whereas the visual recency effect is due to the use of ordinal retrieval cues. Our present hypothesis, therefore, is that visual working memory has similar properties in younger and older children, and that it undergoes surprisingly little, if any, maturational change during this period.

Other workers, using adult subjects, have reported visual recency effects of comparable extent to those reported here, but with rather different methods (Phillips \& Christie, 1977; Walker \& Marshall, 1982; but see Broadbent \& Broadbent, 1981). These results provide further support for the suggestion that there is little developmental change in this aspect of visual working memory. The theoretical interpretation of visual recency in adults is, however, controversial, especially with regard to whether it has a passive basis or involves active visualization (Phillips \& Christie, 1977; Walker \& Marshall, 1982). Recent work on adult subjects has shown that mere exposure to unattended visual inputs can disrupt the use of imagery mnemonics (Logie, 1986), confirming that they gain automatic access to the part of visual working memory that is also involved in active visualization. Further progress in understanding the nature of visual working memory will depend, among many other things, on devising methods whereby its passive and active components can be more clearly separated. If our argument about the early development of automatic processes is correct, then further study of visual memory in children might make a useful contribution.

\section{REFERENCES}

Baddeley, A. D. (1986). Working memory. Oxford: Clarendon Press. BADDELEY, A. D., HrTch, G. J. (1974). Working memory. In G. H. Bower (Ed.), The psychology of learning and motivation (Vol. 8, pp. 47-90). New York: Academic Press.

BARNARD, P. (1985). Interacting cognitive subsystems: A psycholinguistic approach to short-term memory. In A. W. Ellis (Ed.), Progress in the psychology of language (Vol. 2, pp. 197-258). Hillsdale, NJ: Erlbaum.

Broadbent, D. E., \& Broadbent, M. H. P. (1981). Recency effects in visual memory. Quarterly Journal of Experimental Psychology, 33A, 1-15.

Conrad, R. (1971). The chronology of the development of covert speech in children. Developmental Psychology, 5, 398-405.

Dalezman, J. J. (1976). Effects of output order on immediate, delayed and final recall performance. Journal of Experimental Psychology: Human Learning \& Memory, 2, 597-608. 
DREWNOWSKı, A. (1980). Attributes and priorities in short-term recall: A new model of memory span. Journal of Experimental Psychology: General, 109, 208-250.

Dunn, L. M. (1965). Peabody Picture Vocabulary Test. Circle Press, MN: American Guidance Service.

Hitch, G. J., \& Haluday, M. S. (1983). Working memory in children. Philosophical Transactions of the Royal Society London Series B, 302, 325-340.

Hitch, G. J., Halliday, M. S., Schaafstal, A. M., \& Schraagen, J. M. C. (1988). Visual working memory in young children. Memory \& Cognition, 16, 120-132.

KAIL, R. V. (1984). The development of memory in children (2nd ed.). New York: Freeman.

Kosslyn, S. M. (1980). Image and mind. Cambridge, MA: Harvard University Press.

LoGIE, R. H. (1986). Visuo-spatial processing in working memory. Quarterly Journal of Experimental Psychology, 38A, 229-248.

MARTIN, M., \& JoNES, G. V. (1979). Modality dependency of loss of recency in free recall. Psychological Research, 41, 273-289.

MonSELL, S. (1984). Components of working memory underlying verbal skills: A "distributed capacities" view-A tutorial review. In H. Bouma \& D. G. Bouwhuis (Eds.), Attention and performance $X$ (pp. 327-350). Hillsdale, NJ: Erlbaum.

PaIvio, A. (1971). Imagery and verbal processes. New York: Holt, Rinehart and Winston.

Phillips, W. A., \& Christie, D. F. M. (1977). Components of visual memory. Quarterly Journal of Experimental Psychology, 29, 117-133.

Potter, M. C., \& Faulconer, B. A. (1975). Time to understand pictures and words. Nature, 253, 437-438.

SChIANO, D. J., \& WATKINS, M. J. (1981). Speech-like coding of pictures in short-term memory. Memory \& Cognition, 9, 110-114

THORNDIKE, E. L., LORGE, L. (1963). The teacher's word book of 30,000 words. New York: Teacher's College, Columbia University.

Walker, P., \& Marshall, E. (1982). Visual memory and stimulus repetition effects. Journal of Experimental Psychology: General, 111, 348-368.

(Manuscript received January 4, 1988; revision accepted for publication May 24, 1988.) 